\title{
Kikuchi-Fujimoto Disease in an Adolescent Boy
}

\author{
A. Paul Cellura, MD; Stephanie M. Gallitano, MD; Laura E. McDermott, MD, MPH; \\ Rohini Shantharam, MD; Nika Finelt, MD; Sheng Chen, MD, PhD
}

\section{PRACTICE POINTS}

- Kikuchi-Fujimoto disease is an uncommon, self-limited condition characterized by benign lymphadenopathy and variable systemic symptoms.

- Definitive diagnosis is made by excisional lymph node biopsy.

- Treatment options include oral corticosteroids, nonsteroidal anti-inflammatory drugs, antimalarials, and/ or antipyretics.

To the Editor:

Kikuchi-Fujimoto Disease, also called histiocytic necrotizing lymphadenitis, was described in 1972 by both Kikuchi ${ }^{1}$ and Fujimoto et al. ${ }^{2}$ Most cases are reported in Asia, with limited reports in the United States. ${ }^{3-5}$ KikuchiFujimoto disease is a rare, self-limiting condition consisting of benign lymphadenopathy and oftentimes fever and systemic symptoms. Lymph node involvement may mimic non-Hodgkin lymphoma or other reactive lymphadenopathy, rendering diagnostic accuracy challenging. ${ }^{5}$ Cutaneous manifestations are reported in only $16 \%$ to $40 \%$ of patients. ${ }^{6,7}$ Herein, we describe the clinical and pathologic features of a case of Kikuchi-Fujimoto disease with cutaneous involvement in an adolescent boy.

A 13-year-old adolescent boy with no notable medical history presented to the pediatric emergency department with cervical lymphadenopathy, weight loss, intermittent fever, and an evolving rash on the face, ears, arms, and thighs of 6 weeks' duration. The illness began with enlarged lymph nodes and erythematous macules on the face and was diagnosed by his primary care physician as lymphadenitis that was unresponsive to clindamycin. Over the subsequent weeks, the rash worsened, and he developed intermittent fevers, night sweats, abdominal pain, and nausea with a 20 -pound weight loss. He presented to the emergency department 3 weeks prior to the current admission and was noted to have elevated cytomegalovirus (CMV) IgM and IgG in addition to lymphopenia and anemia. He was discharged with outpatient follow-up. The rash progressed to involve the face, ears, arms, and thighs. One day prior to the current admission, the patient's abdominal pain worsened acutely, and he experienced several episodes of emesis. He presented to the pediatric emergency department for further evaluation, and a dermatology consultation was requested at that time.

The patient's rash was asymptomatic. In addition to the above symptoms, he also noted frequent nosebleeds, gingival bleeding, and diffuse myalgia that was most prominent on the hands and feet; he denied diarrhea, sick contacts, recent travel, or insect bites. His vital signs were normal, and he remained afebrile throughout the hospitalization. Physical examination revealed an illappearing patient with sunken eyes and dry lips. He had pink, oval, scaly plaques on the cheeks, ears, and arms (Figure 1). The thighs exhibited folliculocentric erythematous papules. The ocular conjunctivae were clear, but

Drs. Cellura, Shantharam, Finelt, and Chen are from the Department of Dermatology, Hofstra Northwell School of Medicine, New Hyde Park, New York. Drs. Gallitano and McDermott are from the Department of Dermatology, SUNY Downstate Medical Center, Brooklyn, New York. 
white exudative plaques were noted on the tongue. Tender, bilateral, cervical lymphadenopathy and diffuse abdominal tenderness with guarding and hepatosplenomegaly also were present. The fingers and toes were tender upon palpation.

Laboratory workup at admission revealed the following: low white blood cell count, 2700/ $\mu \mathrm{L}$ (reference range, 4500-11,000/ $\mathrm{L}$ ); low hemoglobin, $9.6 \mathrm{~g} / \mathrm{dL}$ (reference range, 14.0-17.5 g/dL); elevated aspartate aminotransferase, $91 \mathrm{U} / \mathrm{L}$ (reference range, 10-30 U/L); and elevated alanine aminotransferase, $118 \mathrm{U} / \mathrm{L}$ (reference range, 10-40 U/L). Lactate dehydrogenase (582 U/L [reference range, 100-200 U/L]), ferritin (1681 ng/mL [reference range, $15-200 \mathrm{ng} / \mathrm{mL}$ ]), and C-reactive protein (6.0 mg/L [reference range, 0.08-3.1 mg/L]) also were elevated. A respiratory viral panel was unremarkable. Blood cultures were negative, and an HIV 1/2 assay was
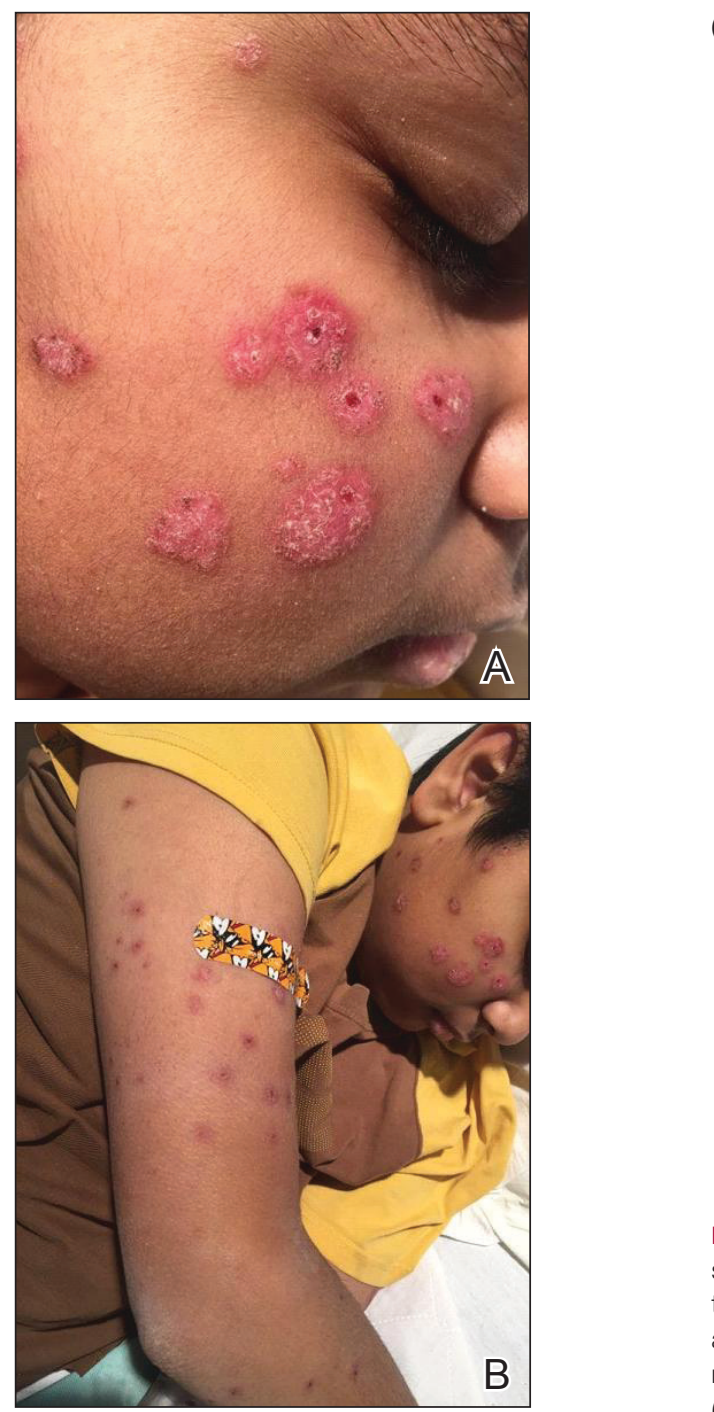

FIGURE 1. A and B, Erythematous scaly plaques of Kikuchi-Fujimoto disease on the right cheek and right upper arm. nonreactive. A chest radiograph demonstrated clear lung fields. Computed tomography of the abdomen and pelvis showed prominent mesenteric, ileocolic, and retroperitoneal lymph nodes.

The differential diagnoses at this time included acute connective tissue disease, a paraneoplastic phenomenon, cutaneous lymphoma, or an infectious etiology. A punch biopsy of the skin as well as tissue cultures were performed from a lesion on the right arm. Quantitative immunoglobulin (IgA, IgG, IgM) levels were checked, all of which were within reference range. An antinuclear antibody (ANA) assay and rheumatoid factor were normal.

The tissue cultures were negative for bacteria, fungi, and mycobacteria. Microscopic examination of the skin biopsy revealed a moderate perivascular and interstitial infiltrate of predominantly histiocytes and lymphocytes with prominent karyorrhectic debris (nuclear dust) in the upper dermis as well as focal vacuolar interface changes with scattered necrotic keratinocytes in the epidermis (Figure 2). Based on these histopathologic findings, a
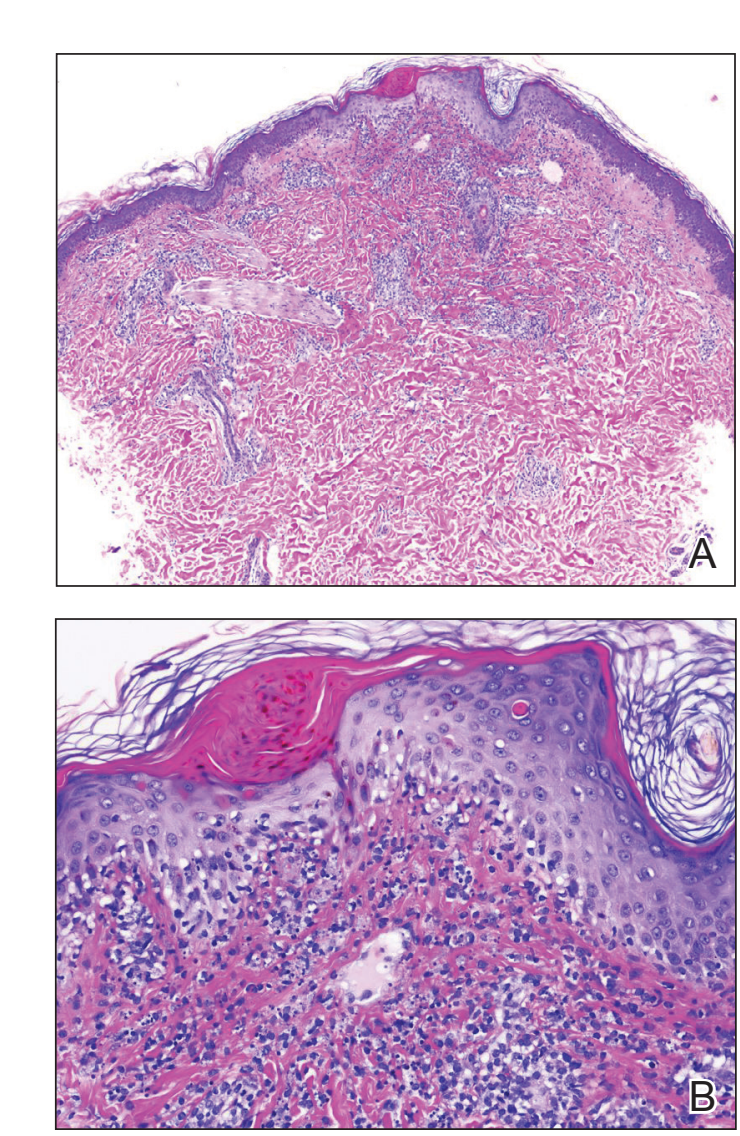

FIGURE 2. A, At low magnification, histology of the skin biopsy showed a moderate perivascular and interstitial infiltrate of inflammatory cells in the upper dermis and focal vacuolar interface changes at the lower epidermis $(\mathrm{H} \& \mathrm{E}$, original magnification $\times 40)$. B, High magnification showed the presence of prominent karyorrhectic debris (nuclear dust) in the dermis and focal vacuolar interface changes with scattered necrotic keratinocytes in the epidermis (H\&E, original magnification $\times 400$ ). 
diagnosis of Kikuchi-Fujimoto disease was considered. To confirm the diagnosis and to rule out the possibility of lymphoma, an excisional biopsy of the cervical lymph node was performed, which showed typical histopathologic features of histiocytic necrotizing lymphadenitis.

Given the patient's clinical presentation with arthralgia, anorexia, lymphadenitis, and hepatosplenomegaly along with histopathologic findings from both the skin and lymph node biopsies, a diagnosis of Kikuchi-Fujimoto disease was made. The patient was conservatively managed with acetaminophen and was discharged with improvement in his appetite and systemic symptoms.

He was seen for follow-up 3 months later in the outpatient clinic. He denied any recurrence of systemic symptoms but endorsed a recent shedding of hair consistent with telogen effluvium. The rash had substantially improved, though residual asymptomatic erythematous plaques remained on the right forehead and right cheek (Figure 3). He was prescribed triamcinolone acetonide cream $0.1 \%$ to apply to the active area twice daily for the following 2 to 3 weeks.

Kikuchi-Fujimoto disease presents with a wide clinical spectrum, classically with benign lymphadenopathy and fever of unknown etiology. 5,6 Lymphadenopathy most often is cervical $(55 \%-99 \%)^{8}$ and unilateral, ${ }^{4,7}$ but patients can present with polyadenopathy $(52 \%){ }^{7,8}$ Constitutional signs commonly include fever $(35 \%-76 \%)$, weight loss, arthritis (5\%-34\%), and leukopenia (25\%-74\%).,4,9

Cutaneous findings have been described in up to $40 \%$ of cases, of which clinical presentation is variable. ${ }^{6}$ Lesions may include blanchable, erythematous, painful, and/or indurated plaques, nodules, or maculopapules with confluence into patches, urticaria, morbilliform lesions, erythema multiforme, eyelid edema, leukocytoclastic vasculitis, papulopustules, ulcerated gingivae, and mucositis. ${ }^{6,7,10-13}$ Patients with skin lesions may be at an increased risk for developing systemic lupus erythematosus (SLE). ${ }^{8}$ Our patient presented with erythematous scaly plaques with a predominance of lesions in photodistributed locations, which clinically mimicked an underlying connective tissue disease process such as SLE.

Infectious agents such as CMV, parvovirus B19, human herpesvirus 6, human herpesvirus 8 and human T-cell lymphotropic virus 1, HIV, Yersinia enterocolitica, and Toxoplasma have all been implicated as possible causes of Kikuchi-Fujimoto disease, but studies have failed to provide convincing causal evidence. $9,14,15$ Our patient had positive IgM and IgG for CMV, which may have incited his disease.

Definitive diagnosis of Kikuchi-Fujimoto disease is made by lymph node excisional biopsy, which histologically exhibits a histiocytic cell proliferation with paracortical foci of necrosis and abundant karyorrhectic debris. ${ }^{5}$ Cutaneous histologic findings that support the diagnosis are variable and may include a dermal histiocytic infiltrate, epidermal change with necrotic keratinocytes, nonneutrophilic karyorrhectic debris, basal vacuolar change,

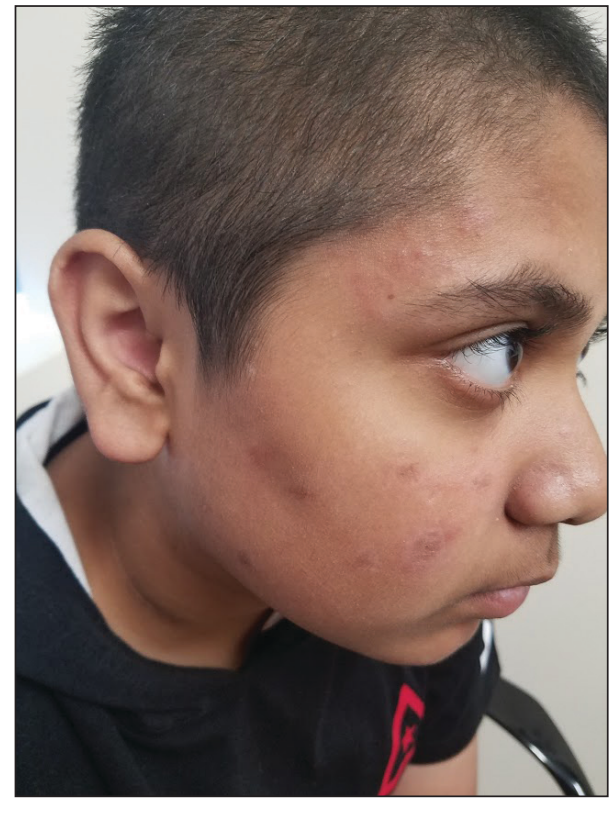

FIGURE 3. Residual plaques of Kikuchi-Fujimoto disease on the right forehead and right cheek with postinflammatory hyperpigmentation on the right cheek.

papillary dermal edema, a nonspecific superficial and deep perivascular infiltrate, and a patchy infiltration of histiocytes and lymphocytes. ${ }^{6,13}$

Clinical and histopathological features of this disease can mimic other diseases, specifically SLE or lymphoma. ${ }^{7}$ An association with SLE has been suspected, though it is not well defined and more frequently is associated with cases from Asia than from Europe $(28 \%$ and 9\%, respectively). ${ }^{9}$ Patients presenting concomitantly with positive ANA, weight loss, arthralgia, and skin lesions are more likely to develop SLE. ${ }^{8}$ Furthermore, the cutaneous histologic finding of interface change suggests a link between the two diseases. As such, recommendations have been made for ANA screenings and follow-up of patients diagnosed with Kikuchi-Fujimoto disease for clinical evidence of autoimmune disease, particularly SLE. ${ }^{6}$ Although our patient did not have a positive ANA, his biopsy did demonstrate interface change, and he should be monitored for possible progression of disease in the future.

Kikuchi-Fujimoto disease differs from lymphoma, as it initially presents with rapid lymph node enlargement as opposed to the gradual enlargement seen in lymphoma. The lymph nodes in Kikuchi-Fujimoto disease often are firm and moveable compared to hard and immobile in lymphoma. ${ }^{3}$ Excisional lymph node biopsy is necessary for both confirming the diagnosis of Kikuchi-Fujimoto disease and ruling out lymphoma. ${ }^{5}$

Spontaneous resolution usually occurs in 1 to 4 months. ${ }^{3,6}$ As such, observation is the most common approach to management. When patients have symptoms 
that limit activities or cause undue distress such as fevers, joint pains, or abdominal pain, systemic treatment options may be desired. Symptomatic treatment can be managed with a short duration of oral corticosteroids, ${ }^{10,11}$ nonsteroidal anti-inflammatory drugs, antimalarials, and/ or antipyretics. ${ }^{8-15}$ There are no guidelines regarding systemic steroid regimens, and various treatment schedules have been successful. Systemic therapy was considered for our patient for his weight loss and abdominal pain; however, by the time of discharge the patient was tolerating oral intake and his abdominal pain had improved.

\section{REFERENCES}

1. Kikuchi M. Lymphadenitis showing focal reticulum cell hyperplasia with nuclear debris and phagocytosis. Nippon Ketsueki Gakkai Zasshi. 1972;35:379-380.

2. Fujimoto Y, Kojima Y, Yamaguchi K. Cervical subacute necrotizing lymphadenitis: a new clinicopathological entity. Naika. 1972; 30:920-927.

3. Feder Jr HM, Liu J, Rezuke WN. Kikuchi disease in Connecticut. J Pediatr. 2014;164:196-200.

4. Kang HM, Kim JY, Choi EH, et al. Clinical characteristics of severe histiocytic necrotizing lymphadenitis (Kikuchi-Fujimoto disease) in children. J Pediatr. 2016;171:208-212.

5. Hutchinson CB, Wang E. Kikuchi-Fujimoto disease. Arch Pathol Lab Med. 2010;134:289-293.
6. Atwater AR, Longly BJ, Aughenbaugh WD. Kikuchi's disease: case report and systematic review of cutaneous and histopathologic presentations. J Am Acad Dermatol. 2008;59:130-136.

7. Yen H-R, Lin P-Y, Chuang W-Y, et al. Skin manifestations of Kikuchi-Fujimoto disease: case report and review. Eur J Pediatr. 2004;163:210-213.

8. Dumas G, Prendki V, Haroche J, et al. Kikuchi-Fujimoto disease: retrospective study of 91 cases and review of literature. Medicine. 2014;93:372-382.

9. Kucukardali Y, Solmazgul E, Kunter E, et al. Kikuchi-Fujimoto disease: analysis of 244 cases. Clin Rheumatol. 2007;26:50-54.

10. Yasukawa K, Matsumura T, Sato-Matsumura KC, et al. Kikuchi's disease and the skin: case report and review of the literature. Br J Dermatol. 2001;144:885-889.

11. Kaur S, Thami GP, Mohan H, et al. Kikuchi disease with facial rash and erythema multiforme. Pediatr Dermatol. 2001;18:403-405.

12. Mauleón C, Valdivielso-Ramos M, Cabeza R, et al. Kikuchi disease with skin lesions mimicking lupus erythematosus. J Dermatol Case Rep. 2012;3:82-85.

13. Obara K, Amoh Y. A case of Kikuchi's disease (histiocytic necrotizing lymphoadenitis) with histiocytic cutaneous involvement. Rheumatol Int. 2015;35:1111-1113

14. Rosado FGN, Tang Y-W, Hasserjian RP, et al. Kikuchi-Fujimoto lymphadenitis: role of parvovirus B-19, Epstein-Barr virus, human herpesvirus 6, and human herpesvirus 8. Hum Pathol. 2013;44:255-259.

15. Chiu $\mathrm{CF}$, Chow $\mathrm{KC}$, Lin $\mathrm{TY}$, et al. Virus infection in patients with histiocytic necrotizing lymphadenitis in Taiwan. detection of Epstein-Barr virus, type I human T-cell lymphotropic virus, and parvovirus B19. Am J Clin Pathol. 2000;113:774-781. 